\title{
The Role of Humoral Agents Released by Platelet Aggregation in the Pathogenesis of Transient Ischemic Attacks
}

\author{
Takao Asano, Akira Tamura, \\ Koji Mil and Keiji SANO \\ Department of Neurosurgery, University of \\ Tokyo Hospital, Tokyo, Japan
}

\begin{abstract}
Summary
For the purpose of investigating the role of the humoral agents released by platelet aggregation in the pathogenesis of transient cerebral ischemic attacks (TIA), the products of platelet suspension which was aggregated by Thrombin was infused as a bolus into the internal carotid artery in rabbits. Before injection, formed elements were filtered off by the use of a milipore filter. The injected material was supposed to contain substances released by platelet aggregation, such as serotonin, histamin, ADP and so on as well as various prostaglandins, among which the existence of thromboxane A2 (TXA $)$ possessing a potent vasoconstricting effect was known.

Before and after the injection, the cortical CBF (COCBF), EEG and systemic arterial pressure (SAP) were continuously monitored under controlled respiration. According to the pattern of response of coCBF following injection of Thrombin-added PRP (T-PRP), a total of 29 rabbits were divided into three groups. In the first group (7/29), the coCBF decreased markedly immediately after the injection, which was accompanied by supression of EEG. Such an ischemic change lasted for 5-10 minutes and then gradually subsided. In two additional rabbits which exhibited the same ischemic pattern, India ink was intravenously administered when the fall of coCBF was at its maximum. This revealed considerable non-filling of the capillary networks of the cerebral cortex in the injected side. The responsible agent for this remarkable cerebral ischemia was considered to be TXA $_{2}$. In the second group (9/29), a biphasic pattern (initial brief ischemia followed by more prolonged hyperemia) was observed. In the third group (13/29), transient but prominent hyperemia was observed. In the two latter groups, there were only slight supressions of EEG as compared to the first group. India ink perfusion of the brain, carried out in the two additional rabbits exhibiting this hyperemic pattern, revealed a patent cerebral microcirculation. This transient hyperemia seen in the second and the third groups was considered to be due to the action of some unknown substance which had a potent vasodilating effect. Recently found Prostacyclin $\left(\mathrm{PGI}_{2}\right)$ was suggested as a possible agent which caused the hyperemic response. The result of the present study is considered to indicate that the dual control system of platelet aggregation and vascular tonus by TXA and Prostacyclin as proposed by Moncada et al. ${ }^{14)}$ is also effective to the cerebral vessels.
\end{abstract}

Key words: Cerebral ischemia, transient. cerebral blood flow, platelet. prostaglandins.

\section{Introduction}

It has been well established that platelet aggregation plays a major role in the pathogenesis of TIA. ${ }^{2}$. ${ }^{21}$ Recent works by Furlow and Bass, ${ }^{8}$ ) or Fieshi et al. ${ }^{11}$ ) have demonstrated also that humoral agents such as arachidonate or ADP infused intraarterially, are capable of producing a focal cerebral ischemic lesion by obstruction of the cerebral microcirculation by platelet aggregates. These evidences suggest that not only the aggregated platelets but also the intravascular humoral agents released in the process of platelet aggregation participate in the pathogenesis of 
TIA. However, the role of humoral agents released by platelet aggregation such as serotonin, histamin, ADP. endoperoxides and its prostaglandin derevatives still remains unknown. Recent discovery of Thromboxane $\mathrm{A}_{2}$ $\left(\mathrm{TXA}_{2}\right.$ ) by Samuelsson et al. ${ }^{10,19)}$ has stimulated interest in the investigation of the role of these humoral agents. $\mathrm{TXA}_{2}$ is produced in the process of platelet aggregation and it has a very potent contracting effect to the artery as well as an aggregatory effect to platelets although its half life is very short, ca. 30 seconds. This vasocontractile capacity of $\mathrm{TXA}_{2}$ was shown to be greatest in the cerebral artery than in the arteries of other organs. ${ }^{61}$ Also it was confirmed that its vasocontractile effect was far more potent than the rest of the humoral agents released by platelet aggregation, such as serotonin or prostaglandin $F_{2 z}$. These findings led to the formation of a hypothesis that platelet aggregation in areas of damaged endothelium can release $\mathrm{TXA}_{2}$ and thus cause constriction of cerebral arteries, which may be relevant to the genesis of TIA or arterial spasms in SAH. ${ }^{61}$

It sometimes occurs, however, that a substance showing a vasocontractile effect in in vitro experiments demonstrates an entirely reverse property in in vivo studies, as known with histamin. ${ }^{17}$ Therefore, it was felt necessary to confirm whether or not any of the substances released by platelet aggregation is at all capable of producing a localized cerebral ischemic lesion simulating human TIA.

In the present study, simultaneous intraarterial injection of the multiple substances released by platelet aggregation was carried out instead of using a single agent. Although such an experiment as the present one would not yield any conclusive result, it may reveal the diversity of the response of a living system and thereby contribute to formation of a working hypothesis as the basis for more analytical studies in the future.

\section{Methods}

1) Preparation of platelet suspension ( $P L S)$ The PLS was prepared according to the method of Shimamoto et al. ${ }^{181}$ Being finally concentrated to a medium containing 1.8 million platelets per cubin $\mathrm{mm}, 1.5 \mathrm{ml}$. of PLS was prewarmed at $37^{\circ} \mathrm{C}$ for 2 minutes. 10 units of Thrombin
(Mochida Co., Ltd.) dissolved in $1 \mathrm{ml}$ of saline was then added into the medium, which caused an immediate aggregation of platelets. The mixture was incubated for 10 seconds, and it was immediately injected as a bolus into the internal carotid artery of an animal through a milipore filter to filter off the formed elements. All of the products released by platelet aggregation was thus injected into the internal carotid artery of the animal.

2) Cannulation of the internal carotid artery More than 30 rabbits were anesthetized with inhalation of Halothane and tracheostomized through a vertical skin incision in the neck. The common, internal and external branches of the right carotid artery were exposed. A fine polyethlene catheter $(0.5 \mathrm{~mm}$. O. D.) was inserted into the lingual artery and the tip of the catheter was advanced towards the orifice of the internal carotid artery. All the branches of the external carotid artery were then ligated so that the material injected through the catheter was completely delivered to the right hemisphere of the brain.

3) Experimental design Following the procedure described above, the animal was fixed in a stereotaxic holder and the skull was exposed. A doubleneedle-type thermocouple (UM Meter, UM2000) was inserted into the right parietal cortex through small burr holes so that the thermistor elements were placed $3 \mathrm{~mm}$. below the brain surface. The reliability of this thermocouple was previously reported. ${ }^{1}$ EEG was recorded by silver-ball electrodes placed epidurally in bilateral fronto-occipital regions. The skull was then covered with a thick cotton pad to avoid fall of the brain temperature. The systemic arterial pressure (SAP) was monitored by cannulation of the femoral artery. The body temperature, being monitored by a rectal thermistor, was maintained between $37^{\circ} \mathrm{C}$ and $38 \mathrm{C}$ by the use of a heating blanket.

The animal was then immobilized by an intravenous injection of Gallamin and was ventilated by a respirator. The arterial blood gases were frequently checked by an autoanalyzer (ABL-1). The normal levels of $\mathrm{pO}_{2}$ and $\mathrm{pCO}_{2}$ was maintained by adjusting the $\mathrm{O}_{2}$ concentration of the inspired air and the tidal volume.

The base deficits were corrected by intravenously administered sodium bicarbonate 
(Meylon). Prior to the injection of thrombinadded PLS (T-PLS), the response of the thermocouple was always checked by injection of small amounts of saline and/or transient apnea. The T-PLS was injected when EEG returned to almost the normal pattern.

Following an injection, SAP, EEG, and the cortical CBF (CoCBF) as measured by the thermocouple, were continuously monitored for 30 minutes in 29 rabbits, which were then sacrificed by venous injection of saturated pentobarbital solution. The brain was removed and fixed in 10 percent formalin. The specimens were examined with Hematoxylin-Eosin, Klüver-Barrerra and periodic acid-Schiff stains.

In an additional five rabbits which underwent the same procedure and the injection of T-PLS as above, $30-40 \mathrm{ml}$ of India ink (Pelikan biological ink) was injected intravenously when the change of coCBF was maximal. The animals were then sacrificed and the brain was removed. In addition to the routine histological studies as described above, the status of the cerebral microcirculation was studied in this group with 100 micra sections.

\section{Results}

A transient, mild increase of coCBF was observed at separate test injections of PLS and thrombin, which was similar to the pattern observed at injection of saline. EEG revealed no significant changes during and after these procedures.

The injection of T-PLS resulted in remarkable changes both in coCBF and EEG. According to the patterns of changes in coCBF, the animals were divided into three groups. In the first group ( 7 of 29 total rabbits), marked decrease of coCBF took place immediately after injection of T-PLS. (Fig. 1). The decrease of coCBF was always accompanied by various forms of transient $E E G$ supression, such as low voltage, slowing of the basal rhythm, appearance of high amplitude slow waves and/or complete flattening. Changes of SAP were also observed immediately after injection of T-PLS. The effect of the changes in SAP on the cerebral circulation was considered insignificant because they were only mild and very short-lasting. The changes in coCBF and in EEG lasted for 5-10 minutes and they gradually returned to the preinjection status. In histological examinations, no significant changes
ISCHEMIA PATTERN
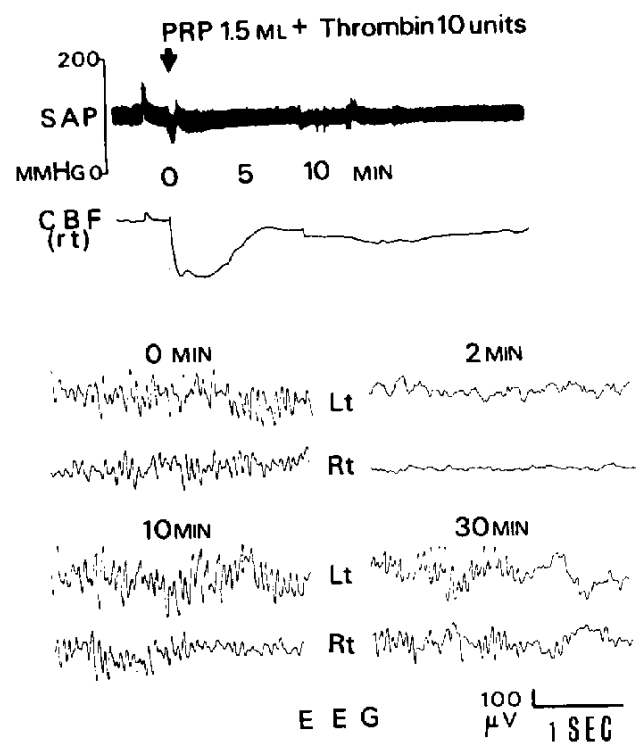

Fig. 1 Immediately after the injection of T-PLS, the coCBF decreased close to the zero level for about three minutes. Extreme low voltage and transient flattening was seen. These changes of coCBF and EEG gradually recovered in twenty minutes.

were found in the cortical structure.

Two of the five additional rabbits also exhibited the sudden fall of coCBF following injections of T-PLS as in the first group. Injection of India ink was carried out when the coCBF decreased maximally, i. e., about 5 minutes after the injection of T-PLS. The autopsied brain revealed complete non-filling of the right hemisphere in one animal and a partial nonfilling in the right parietal region in the other. (Fig. 2A) Complete non-filling of the cortical capillary networks was confirmed by 100 micra sections of the specimens taken from the area of macroscopical non-filling. This area of nonfilling was thoroughly examined in serial sections with PAS-stained materials. This revealed almost empty lumina of the arterioles and the capillary networks. Platelet thrombi were only rarely found, (Fig. 2B) These histological findings indicate that the capillary networks of the area of non-filling was almost devoid of formed elements, such as platelet thrombi or red cell aggregates.

In the second group of animals $(9 / 29)$, the change of coCBF was biphasic, i.e., the initial 


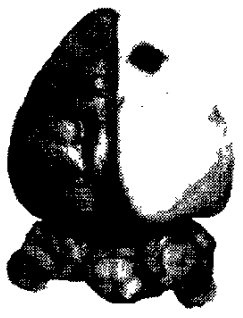

A

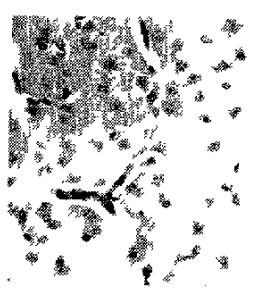

B
Fig. 2-A. The external appearance of the brain perfused with carbon black. Note the complete non-filling of the right hemisphere.

2-B. The histological specimen taken from the cortical area of the macroscopical non-filling in the above brain. (PAS stain).

The lumina of the capillarties are almost empty without recognizable platelet and/or red cell aggregates.

ischemia was immediately followed by hyperemia. (Fig. 3) The duration of the initial sudden decrease of coCBF was very transient and it was rapidly followed by pronounced increase of coCBF. This hyperemia persisted for 5-10 minutes and gradually returned to the original level. In EEG, similar changes as in the first group were observed. The frequency of EEG supression, however, was definitely less than in the first group. Only one of the five additional rabbits exhibited this biphasic pattern of coCBF. No perfusion defects were found in the autopsied brain.

In the third group of animals (13/29), sudden and pronounced increase of coCBF took place immediately after an injection of T-PLS. (Fig. 4) The increase of coCBF persisted for 5-10 minutes and gradually fell to the preinjection level. In seven of thirteen animals of this group, no change in EEG was observed during and after the injection of T-PLS. In the rest of the group, only transient low voltage or slowing of the basal rhythms were observed. Two of the five additional rabbits showed a remarkable increase of coCBF as in the third group. Venous injection of India ink carried out at the height of increase in $\mathrm{CoCBF}$, revealed no perfusion defects. Histologically, no pathological findings were revealed both in the second and the third groups.

Summary of the experimental result is repre-

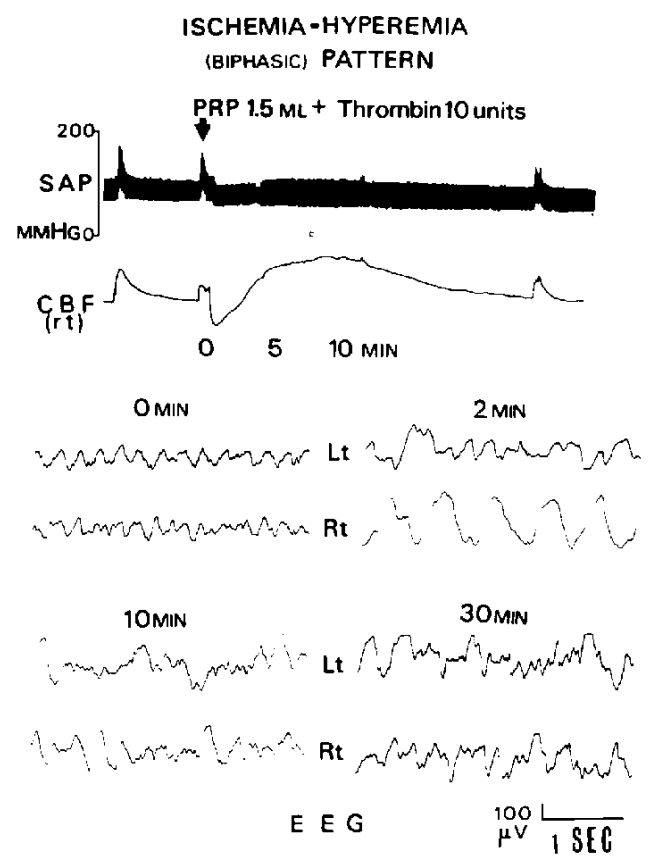

Fig. 3 The biphasic pattern of coCBF. Note the transient increase of $\operatorname{coCBF}$ which were parallel to the spontaneous changes of SAP. The SAP changed little during and after the injection of $T$ PLS.

sentatively shown in the Fig. 5. As shown in this figure, the changes of EEG were most severe in the first group and became less frequent in the second and the third groups. It may be said that the supression of EEG obtained in the present study reflected the severity of decrease in coCBF.

\section{Discussion}

The rationale of the present experiment. The pathogenetic importance of platelet aggregation in TIA seems well established today. ${ }^{2}, 4,12,21$ ) The platelets which are aggregated at the atheromatous plaques in the neck vessels and transported by the blood stream to the cerebral vasculature, are thought to cause focal embolic phenomena. Such a platelet embolus was found occasionally in the retinal arteries in patients sustaining TIAs. ${ }^{7}$ Experimentally, focal cerebral ischemia accompanied by supression of EEG was produced in animals by intracarotid infusions of ADP or sodium arachidonate which are known to possess an aggregatory effect to platelets. Obliteration of the cerebral capillary 
HYPEREMIA PATTERN
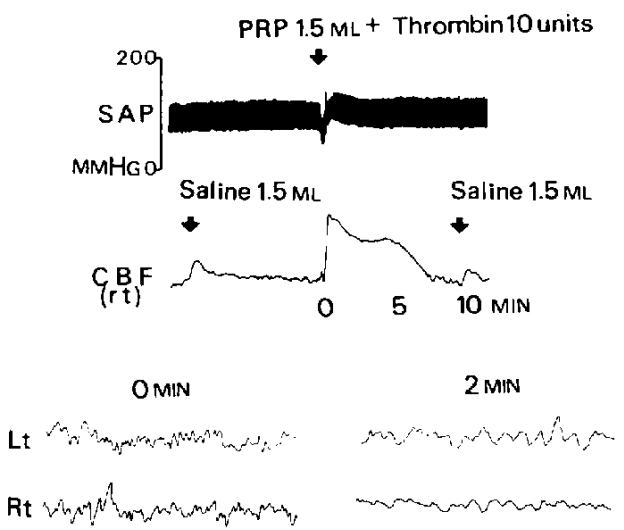

$10 \mathrm{MIN}$

30 MIN

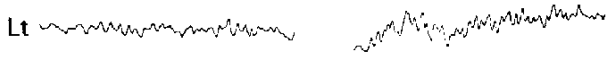

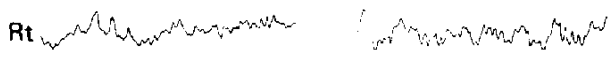

EEG $\quad \stackrel{100}{\mu V} \longleftarrow$

Fig. 4 The first small peak of coCBF is the usual response to intracarotid injection of saline, PLS or thrombin alone. Note the prominent increase of coCBF after injection of T-PLS. In this animal, definite slowing and low voltage in EEG were observed.
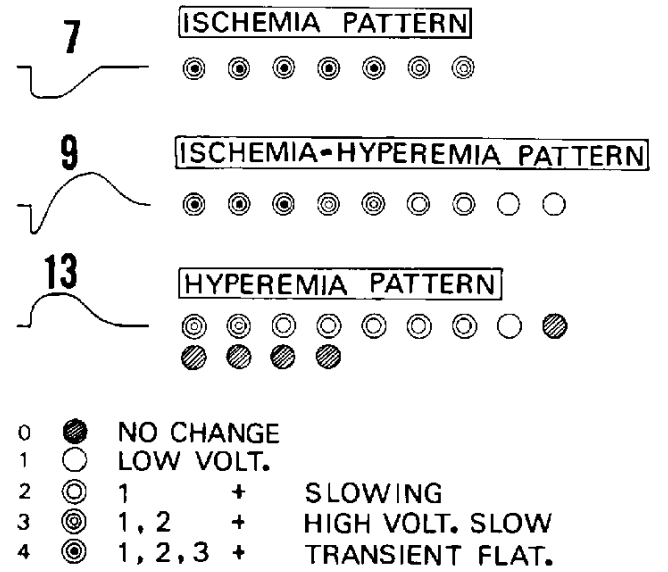

Fig. 5 Summary of the changes of coCBF and EEG. It is clearly shown that the degree of supression of EEG is paralell to the severity of decrease in coCBF. The transient flattening of EEG was arbitrarily defined as an extreme low voltage less than 10 microvolt lasting more than three seconds. networks by platelet plugs was demonstrated with the histological specimens in these studies. ${ }^{8111}$ Such clinical as well as experimental data reinforced the view that TIA is caused by the aggregated platelets which act as embolus in the arterioles or in the arterial side of the capillary networks of the brain.

The platelets, however, release various substances in the process of aggregation, such as serotonin, ADP, histamin, norepinephrine, multiple products of prostaglandins, etc. ${ }^{20}$ As some of these substances are known to possess vasoactive effects, it is easily speculated that these agents released by platelet aggregation may induce secondary changes in the cerebral vessels. If arterial constriction is brought about, for example, it will cause a more widespread and severe cerebral ischemia than in the case of simple arteriolar obstructions by platelet aggregates. Therefore, it seemed worthwhile to investigate the pathogenetic role of the humoral agents released by platelet aggregation in the genesis of TIA, which may enable development of a better form of preventive therapies in the future.

In a previous experiment reported elsewhere by Shimamoto et al., ${ }^{8)}$ it was shown that $\mathrm{TXA}_{2}$ was liberated by aggregation of platelet being induced by thrombin.

In that experiment, antagonists to serotonin, histamin and norepinephrine as well as indomethacine which blocked formation of other prostaglandins were added to the PLS prior to addition of thrombin. Therefore, the vasoconstrictive effect of the T-PLS demonstrated in that study was considered to be solely due to released $\mathrm{TXA}_{2}$ and/or its precursors, i.e., the endoperoxides such as $\mathbf{P G G}_{2}$ and $\mathbf{P G H}_{2}$. In the present study, those antagonists to various agents were not used in view of avoiding their possible interactions. Therefore, all the substances released by platelet aggregation, such as serotonin, NE, ADP, histamin and various prostaglandins including $\mathrm{TXA}_{2}$ were simultaneously injected into the internal carotid artery. As initially stated, it is clear that such an experiment as the present one, will not yield any definitive conclusions as to the mechanism of TIA. The purpose of the present study, however, was to verify the hypothesis that the humoral agents released by platelet aggregation, no matter what they may be, were capable of producing 
cerebral ischemic lesions mimicking the human TIA. In this respect, a very interesting result was obtained which would necessitate revision of the initial hypothesis. In the following paragraphs. the result of the present study will be discussed in more detail, finally to suggest a new working hypothesis concerning the existence of a dual control system of vascular tonus and platelet aggregation.

Patterns of $\operatorname{COCBF}$ and their implications. In the first ischemic pattern (Fig. 1), the decrease of coCBF was severe enough to produce changes of EEG such as transient flattening and so on in the majority of animals. This transient reduction of coCBF associated with temporary suppression of EEG is considered to simulate human TIAs. In contrast to the previous experiments using ADP or sodium arachidonate, obliteration of the capillary networks by platelet aggregates was not found through careful survey of histological specimens in the present study. This may indicate that the reduction of $\mathrm{COCBF}$ in this group was due to a generalized constriction of the cerebral vessels by some potent vasocontractile agent rather than to formation of platelet aggregates. In this regard, the role of $\mathrm{TXA}_{2}$ as the major causative agent is suggested because it is unlikely that other substances such as serotonin. NE or histamin would cause such a pronounced reduction of CBF by single carotid injection as in the present study. ${ }^{13,15,16)}$

Participation of other vasoactive forms of prostaglandins, such as $\mathrm{PGF}_{2 x}$ or $\mathrm{PGE}_{2}$, is also unlikely because it is now known that the conversion of the endoperoxides $\left(\mathrm{PGG}_{2}\right.$ and $\mathrm{PGH}_{2}$ ) to the thromboxanes is the preferential metabolic pathway in platelets. ${ }^{6}{ }^{19)}$ As initially expected, anyway, this ischemic episode of the brain demonstrated in the first experimental group is considered to be a supportive evidence to the hypothesis that the agents released by platelet aggregation are capable of causing stroke.

The hyperemic responses of the cerebral vessel observed in the second and the third experimental groups were confusing at first as they were quite the contrary to what was initially anticipated. The rapid and prominent increase of coCBF, preservation of the cerebral microcirculation as shown in the brains perfused with carbon black and paucity of supressions of EEG. all indicated that a real increase of coCBF occurred by the action of some potent vasodilating substance. Among the substances released by platelet aggregation, none were known to exhibit such a potent vasodilating effect when injected into the internal carotid artery. ${ }^{13,15,16,17)}$ Therefore, existence of some unknown agent possessing a potent vasodilating effect had to be postulated to explain the present result.

In this regard, the new hypothesis of dual control system on vascular tonus as well as platelet aggregation which was recently suggested by Moncada et al. ${ }^{5.14}$ seems very interesting. They identified a new prostaglandin $\left(\mathrm{PGI}_{2}\right.$, or Prostacyclin) which was derived from arachidonic acid through the endoperoxide, $\mathrm{PGH}_{2}{ }^{\left.{ }^{9},{ }^{10}\right)}$ The conversion from $\mathrm{PGH}_{2}$ to $\mathrm{PGI}_{2}$ was accomplished by a microsomal preparation of arterial walls. ${ }^{13}$ The unique property of $\mathrm{PGI}_{2}$ is that it has a potent vasodilatatory as well as antiaggregatory effect both of which are antag-

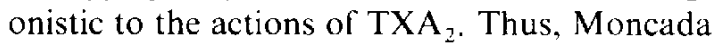
et al. $^{3,14)}$ have proposed that a balance between the thromboxane generating system in platelets and the prostacyclin generating system in vascular endothelium regulates platelet aggregation. Further, based on studies using bovine aortic strips, Dusting et al. " has enlarged this concept to include the tonus of arterial walls. According to this hypothesis, platelets when stimulated to aggregate, produce $\mathrm{PGH}_{2}$. This endoperoxide can either be converted to $\mathrm{TXA}_{2}$, which initiates vasoconstriction, and a platelet aggregation that is associated with an inhibition of cAMP accumulation, or escape the platelet and be converted to $\mathrm{PGI}_{2}$ by the vessel wall. ${ }^{9}$ (Fig. 6). $\mathrm{PGI}_{2}$ can then dilate the vessel and stimulate platelet adenylate cyclase which will inhibit the aggregation and platelet thrombus formation that has been induced by the endoperoxide-TXA $\mathrm{A}_{2}$ system.

If it is assumed that the postulated vasodilator in the present study is $\mathrm{PGI}_{2}$, then a clear explanation of the present result based on the above hypothesis can be given as follows. The TPLS releases both $\mathrm{TXA}_{2}$ and its precursor, $\mathrm{PGH}_{2}$. When the conversion from $\mathrm{PGH}_{2}$ to TXA $_{2}$ is by far preponderant over the conversion from $\mathrm{PGH}_{2}$ to $\mathrm{PGI}_{2}$, a vasoconstriction as in the first experimental group takes place. Conversely, a vasodilation will be brought about when the convertion of $\mathrm{PGH}_{2}$ to $\mathrm{PGI}_{2}$ is more pre- 


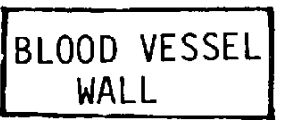

PLATELET

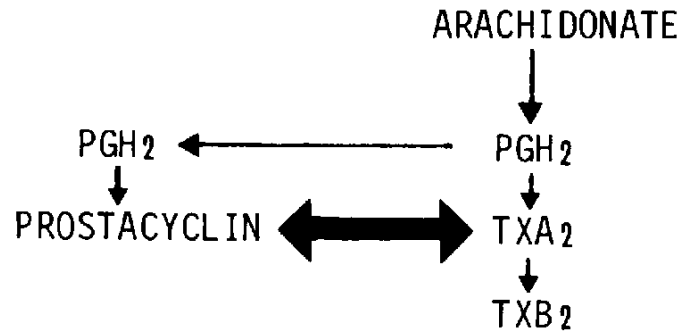

Fig. 6 The routes of systhesis of $\mathrm{TXA}_{2}$ and $\mathrm{PGI}_{2}$ (prostacyclin). In the process of aggregation of platelets. $\mathrm{PGH}_{2}$ (and $-\mathrm{G}_{2}$ ) is liberated in and outside the platelet. This $\mathrm{PGH}_{2}$ is converted to TXA $_{2}$, by the action of enzymes in the microsomāl fraction of platelets. The free $\mathrm{PGH}_{2}$, discharged into the blood stream. is conveyed to the cerebral vessel wall, where it is converted to $\mathrm{PGI}_{2}$. The antagonistic effects of $\mathrm{TXA}_{2}$ and $\mathrm{PGI}_{2}$ in terms of vascular tonus and platelet aggregation are expressed by the large arrow.

ponderant, as shown in the second and the third experimental group. In addition, $\mathrm{PGH}_{2}$ was reported to cause a biphasic response of vascular smooth muscles (initial constriction followed by dilation), which was very similar to the biphasic pattern of coCBF as seen in the second experimental group of the present experiment, especially in terms of their time-courses. ${ }^{5}$

Such a clear parallelism between an experimental result and a hypothesis seems to implicate more than coincidence. Or, the result of the present experiment might be interpreted as an indirect evidence that the dual control system of $\mathrm{TXA}_{2}$ and $\mathrm{PGI}_{2}$ is also effective to the cerebral vessels.

The initial purpose of the present study, i.e., to look for an effective hypothesis in regard to the pathogenesis of TIA, thus scems to have been accomplished. This working hypothesis, in turn, awaits confirmations through further studies.

\section{Acknowledgement}

This work is dedicated to the memory of late professor Takio Shimamoto. We are very grateful to his colleagues, Drs. Yoshimi Takashima, Masahiko Kobayashi and Takeo Takahashi for their assistance in the preparation of the plateletsuspension used in this study.

\section{References}

1) Asano, T. and Sano, K. : Pathogenetic role of noreflow phenomenon in experimental subarachnoid hemorrhage in dogs. J. Neurosurg. 46: $454-466,1977$

2) Barnett, H. J. M.: Pathogenesis of transient ischemic attacks, in Cerebrovascular diseases. Tenth Princeton Conference. Scheinberg, P. (ed). Raven Press, New York, pp. 1-22, 1976

3) Bunting S., Gryglewski R.. Moncada S., and Vane J. R.: Arterial walls generate from prostaglandin endoperoxides a substance (prostaglandin $\mathrm{X}$ ) which relaxes strip of mesenteric and coeliac arteries and inhibits platelet aggregatin. Prostaglandins 12: 897-913, 1976

4) Denton I. C., Robertson J. T., and Dugdale M. : An assessment of early platelet activity in experimental subarachnoid hemorrhage and middle cerebral artery thrombosis in the cat. Stroke 2: 268-272, 1971

5) Dusting G. J., Moncada S., and Vane J. R.: Prostaglandin (PGX) is the endogenous metabolite responsible for relaxation of coronary arteries induced by arachidonic acid. Prostaglandins 13: 3-15, 1977

6) Ellis, E. F., Nies A. S., and Oates J. A. : Cerebral arterial smooth muscle contraction by Thromboxane A2. Stroke 8: 480-483, 1977

7) Fisher C. M. : Observations of the fundus oculi in trancient monocular blindness. Neurology 9: 333-347, 1959

8) Furlow T. W. and Bass N. H. : Stroke in rats produced by carotid injection of sodium arachidonate. Science 187: 658-660, 1974

9) Gorman R., Bunting S., and Miller O.: Modulation of human platelet adenylate cyclase by prostacyclin (PGX). Prostaglandins 13: 377-388, 1977

10) Hamberg M., Svensson J., and Samuelsson B. : Thromboxanes: a new group of biologically active compounds derived from prostaglandin endoperoxides. Proc. Nat. Acad. Sci. USA. 72: 2994-2998, 1975

11) Heuser D., Fieshi C., and Volante F. : Platelet emboli and focal cerebral ischemia. An experimental study on the circulatory and metabolic effects of intracarotid infusion of ADP and arachidonic acid in rabbits. in The cerebral vessel wall. Cervos-Navarro (ed). Raven Press. New York, 1976. pp. 149-156

12) Kalendorsky Z, Austin J., and Steele P.: 
Increased platelet aggregability in young patients with stroke. Arch Neurol. 32: 13-20, 1975

13) Mendelow A. D., Eidelman B. H., McCalden T. A., and Rosendorff C.: Cerebrovascular responses to infused 5-hydrotryptamin in the baboon. Stroke 8: 322 325, 1977

14) Moncada S., Gryglewski R., and Bunting S. : An enzyme isolated from arteries transforms prostaglandin endoperoxides to an unstable substance that inhibits platelet aggregation. Nature 263: 663-665, 1976

15) Olesen $\mathrm{J}_{\text {. }}$ : The effect of intracarotid epinephrine, norepinephrine and angiotensin on the regional cerebral blood flow in man. Neurology 22: 978-987, 1972

16) Olesen J.: Cerebral blood flow. Methods for measurements, regulation, effects of drugs and changes in disease. Acta neurologica scand. supple 57, 50: 48-53, 1974

17) Purves M. J.: The physiology of the cerebral circulation. Cambridge Univ Press. 1972. pp. 349

18) Shimamoto T., Takashima Y., Kobayashi M., Moriya K., and Takahashi Y.: A Thromboxane A2-antagonistic effect of pyridinolcarbamate and phthalazinol. Proc. Jap. Acad. 52: 591-594, 1976

19) Svensson J., Hamberg M., Samuelsson B. : On the qormation and effects of Thromboxane $\mathrm{A}_{2}$ in human platelets. Acta physiol. scand. 98: 285-294, 1976

20) Weiss H. J.: Platelet physiology and abnormalities of platelet function. New Eng. $J$. Med. 293: 531-541, 1975

21) Yatsu F.: Editorial. Stroke therapy: status of anti-platelet aggregation drugs. Neurology 27: 503-504, 1977 